\title{
\begin{tabular}{l|l} 
Mitraries & DSpace@MIT
\end{tabular}
}

\author{
MIT Open Access Articles
}

\section{Vibrational Analysis of Semicrystalline Polyethylene Using Molecular Dynamics Simulation}

The MIT Faculty has made this article openly available. Please share how this access benefits you. Your story matters.

Citation: Brayton, Alexander L. et al., "Vibrational Analysis of Semicrystalline Polyethylene Using Molecular Dynamics Simulation." Macromolecules 50, 17 (September 2017): 6690-6701 (c)2017 Authors

As Published: https://dx.doi.org/10.1021/ACS.MACROMOL.7B00995

Publisher: American Chemical Society (ACS)

Persistent URL: https://hdl.handle.net/1721.1/130131

Version: Author's final manuscript: final author's manuscript post peer review, without publisher's formatting or copy editing

Terms of Use: Article is made available in accordance with the publisher's policy and may be subject to US copyright law. Please refer to the publisher's site for terms of use. 


\title{
Vibrational Analysis of Semicrystalline Polyethylene Using Molecular Dynamics Simulation
}

\author{
Alexander L. Brayton, ${ }^{\dagger}$ In-Chul Yeh, ${ }^{\ddagger \odot}$ Jan W. Andzelm, ${ }^{\ddagger \odot}$ and Gregory C. Rutledge, ${ }^{\star}{ }^{\dagger} \odot$ \\ ${ }^{\dagger}$ Department of Chemical Engineering, Massachusetts Institute of Technology, 77 Massachusetts Avenue, Cambridge, Massachusetts \\ 02139, United States \\ ${ }^{\ddagger}$ U.S. Army Research Laboratory, Aberdeen Proving Ground, Maryland 21005, United States
}

\section{Supporting Information}

ABSTRACT: The vibrational spectra of semicrystalline polyethylene and its distinct domains were investigated using molecular dynamics (MD) simulations. A method for the vibrational analysis of the domains within the lamellar stack model of semicrystalline polymers has been developed and demonstrated on semicrystalline polyethylene using force fields having either united atom (UA) or explicit atom (EA) detail. In the UA description, the calculated vibrational spectra were found to differ from the observed skeletal vibrations of polyethylene with
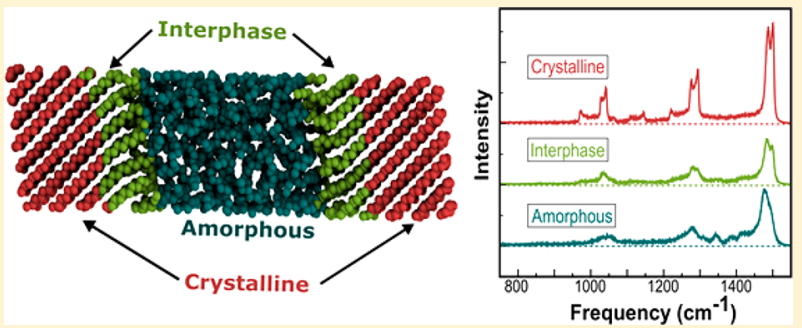
the force field used in this work. Therefore, a modified UA force field with different stretching and bending force constants is proposed, which was found to reproduce the observed frequencies of the skeletal vibrations. In the EA description, the vibrational spectra of semicrystalline polyethylene were in satisfactory agreement with typical infrared and Raman signatures of polyethylene melts and crystals. Experimental interpretations regarding the assignment of peaks in the Raman spectra to components of semicrystalline polyethylene were examined. The spectrum of the interphase domain obtained using the EA model was found to be adequately reproduced by a superposition of the spectra of the crystalline and amorphous domains, at variance with experimental observation. The lack of a distinct interphase spectrum in the EA model was attributed to the absence of the $\mathrm{CH}_{2}$ bending peak associated with the orthorhombic phase, despite confirming an orthorhombic crystal structure in the crystalline domain.

\section{INTRODUCTION}

Semicrystalline (SC) polymers are so-called because they consist of crystalline regions, wherein chain segments are typically organized parallel in lamellar crystallites, interspersed with amorphous regions, wherein segments with meltlike chain conformations and packing are observed. The prototypical SC polymer is polyethylene (PE). A simplified schematic of semicrystalline polyethylene (SCPE) is depicted in Figure 1a. The simplest structural motif in these materials is the lamellar stack, formed by alternating crystalline lamellae and noncrystalline domains. ${ }^{1}$ The noncrystalline domain can be divided further into an amorphous region and an interfacial region between the crystal and amorphous domains that is constrained by the lamellae. The interfacial region has been extensively discussed in the literature and has been described variously as the transition layer ${ }^{2}$ or rigid amorphous domain, ${ }^{3}$ having hindered-rotational, ${ }^{4}$ noncrystalline consecutive trans, ${ }^{5}$ or alltrans conformations lacking lateral order. ${ }^{6}$ In the current context, we employ the term "interphase", introduced originally by Flory and co-workers, ${ }^{7}$ to identify material that is intermediate between crystalline and amorphous. When polymer chain lengths greatly exceed the length of the lamellar thickness, which varies between 5 and $50 \mathrm{~nm}$ for PE, unique topological features (i.e., tails, bridges, and loops) can occur as chains exit and re-enter the same or different lamellae. These structural features of the interlamellar region are illustrated in Figure $1 b$.

Despite its simple chemical structure (composed of bonded methylene groups), the structural details in SCPE still remain an area of active research, especially those concerning the interfacial region, whose structure is crucial to the transfer of mechanical stress from one domain to another. However, because it is thin and always coexists with the larger crystalline and amorphous domains, it remains elusive to direct experimental study. ${ }^{8-11}$ Hence, even though SCPE is chemically simple, it is structurally heterogeneous, consisting of several interconnected domains, which considerably complicates the interpretation of data collected from such materials. An understanding of the properties of the constitutive domains has proven useful in building models to describe the material's bulk behavior. For example, the mechanical properties of the individual domains (including interphase) have been used to predict the overall mechanical response of SCPE using micromechanical modeling. ${ }^{9,10}$ However, with regard to the dynamics of SC polymers, the properties of the individual domains have not been fully characterized.

Received: May 12, 2017

Revised: $\quad$ August 1, 2017 
(a)

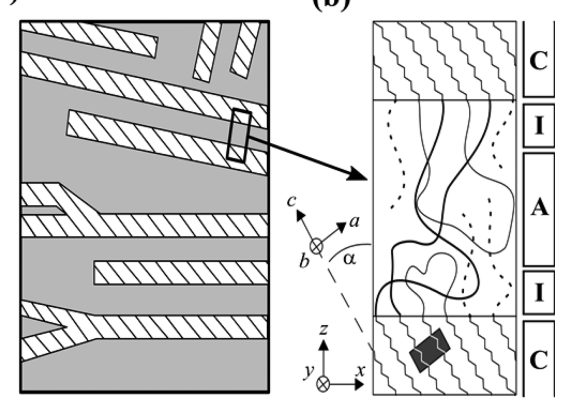

Figure 1. (a) Illustration of a lamellar morphology of SCPE composed of the crystalline lamellae and the noncrystalline material as represented by the gray area. (b) Schematic of the lamellar stack and its topological features, where loops are denoted by thin solid lines, tails by dashed lines, and bridges by bold solid lines. The unit cell is depicted by the gray box with principal axes $(a, b, c)$ tilted by an angle $\alpha$ with respect to the Cartesian $z$-axis. The labels "C", "I", and "A" denote the crystalline, interphase, and amorphous domains, respectively.

Just as semicrystalline polymers are structurally heterogeneous, they are also dynamically heterogeneous due to the differences in dynamical behavior associated with different structures. When characterized macroscopically, the dynamic behavior observed is a composite of the dynamical behaviors of the spatially and structurally distinct domains as well as their interdependences. Additionally, dynamic phenomena in polymer systems are distributed over a wide spectrum of time and length scales, where fast dynamic events such as bond vibrations occur on the order of femtoseconds and angstroms, while slow events such as the lamellar thickening occur on the order of days and micrometers. The fast motions are readily accessible to investigation using molecular simulation.

The vibrational motions of a molecule can be summarized through its vibrational spectrum, which quantifies the distribution of vibration frequencies in frequency space. Experimentally, the vibrational spectrum of a material is characterized through infrared or Raman spectroscopy techniques; the total spectrum comprises modes that are infrared-active, associated with temporally varying dipole moment, modes that are Raman-active, associated with temporally varying polarization, and modes that are inactive with respect to either technique. The vibrational spectrum can also be computed; one technique to do so is normal-mode analysis. ${ }^{12}$ A vibrational normal mode (NM) refers to the independent motions of all or a subset of atoms in the system, which oscillate at a characteristic frequency (CF) ${ }^{13}$ At low temperature, the vibrational NM's can be obtained within the harmonic approximation through diagonalization of the matrix of second derivatives of the potential energy surface (PES) with respect to atom displacements, widely referred to as the Hessian matrix; ${ }^{12}$ the square roots of the eigenvalues of the Hessian matrix give the CF's, and the eigenvectors describe the displacement of atoms in each mode. ${ }^{12}$ In the harmonic approximation, the vibrational spectrum, denoted here as $P(v)$, is given by $P_{\text {harm }}(v)=\sum_{j=1}^{3 N} \delta\left(v-v_{j}\right)$, where $N$ is the number of atoms, $\delta$ indicates the Dirac delta function, $v$ is the frequency, and $v_{j}$ is the CF of the jth mode. At finite temperatures, the harmonic approximation begins to break down. ${ }^{12,14,15}$ In these cases, vibrational NM's can be computed using methods that employ a quasiharmonic approximation. ${ }^{14,16,17}$ A self-consistent quasiharmonic approximation was previously employed by our group to compute the temperature-dependent thermomechanical properties of crystalline PE. ${ }^{18}$

The vibrational spectra of $\mathrm{PE}$ have been the focus of extensive study using both experimental ${ }^{6,19-27}$ and computational $^{28-30}$ techniques. While much of this attention has been focused on the spectra of the crystalline and amorphous domains, a few studies have discussed the vibrational signature of the interfacial region, ${ }^{21,31-33}$ all of which are related to the seminal study of Strobl and Hagedorn. ${ }^{6}$ Strobl and Hagedorn ${ }^{6}$ proposed a method to analyze the Raman spectrum of SCPE to estimate the relative composition of the ordered crystalline domain, disordered amorphous domain, and an interfacial region of intermediate order. They identified the spectral peak of the methylene $\left(\mathrm{CH}_{2}\right)$ bending mode near $1416 \mathrm{~cm}^{-1}$, which arises from correlation splitting in the orthorhombic crystal structure, ${ }^{20}$ as a good estimator of crystallinity. To estimate the amorphous content, they used the broad peak of the skeletal stretching mode near $1080 \mathrm{~cm}^{-1}$. Furthermore, Strobl and Hagedorn found that the superposition of melt and crystal-like Raman spectra failed to capture a change in relative peak heights in the $\mathrm{CH}_{2}$ bending region of the SC spectrum. To account for this change, they proposed a third domain occurring between the amorphous and crystalline domains with chain segments of all-trans character without lateral order. The proposed interfacial domain is similar to the rotator phase of $n$-alkanes, which exhibits a single bending peak near 1438 $\mathrm{cm}^{-1}$ without the additional $1416 \mathrm{~cm}^{-1}$ peak observed in the orthorhombic phase. ${ }^{20}$ A rotator-like, interfacial domain could account for the change in relative peak intensities observed in the bending regime of the SCPE spectrum. Glotin and Mandelkern ${ }^{24}$ provided extensive testing of the method of Strobl and Hagedorn for a variety of PE morphologies and found excellent agreement between the results of this method and complementary techniques to estimate the relative composition of PE. Additionally, there has been discussion on the accuracy of the method of Strobl and Hagedorn. ${ }^{32,34}$ Naylor et al. ${ }^{32}$ argued that the error associated with the peak fitting procedure was similar in magnitude to the interfacial content itself, and the estimation of the interfacial content using the Raman spectrum of SCPE could be seriously questioned based on their findings. In a follow-up to Naylor et al., Mandelkern and Alamo ${ }^{34}$ commented that estimates of interfacial composition using the method of Strobl and Hagedorn were consistent with estimates from different experimental techniques. Even nearly four decades later, the method of Strobl and Hagedorn is still used to characterize the domain compositions of $\mathrm{PE}^{5,35,36}$ and has been extended to additional SC polymers. ${ }^{37}$

In this work, we investigate the dynamic heterogeneities associated with the vibrational motions within SCPE using molecular dynamics (MD) simulations. For the first time, we present the vibrational signature for realistic configurations of SCPE, calculated using molecular simulation. Furthermore, we present the "subspectra" of the distinct domains within SCPE. To do so, we employ the unique resolution offered by molecular simulation, which allows us to restrict our vibrational analysis to atoms within specific domains of interest. Using these results, we examine the details of the calculated vibrational spectra and compare our results to Strobl and Hagedorn. Most notably, we can calculate the spectrum of the interphase domain directly, which was suggested by Strobl and Hagedorn to be uniquely characterized by a single $\mathrm{CH}_{2}$ bending peak near $1438 \mathrm{~cm}^{-1}$. We sought to confirm this observation 
through simulation and identify additional salient features of the vibrational spectrum of the interphase, which remains difficult to characterize experimentally.

\section{MODEL AND SIMULATION METHODOLOGY}

2.1. Semicrystalline Polyethylene (SCPE) Model. The SCPE configurations studied in this work were generated using the Enhanced Monte Carlo (EMC) software, ${ }^{38}$ which implements the Interphase Monte Carlo (IMC) method reported by Rutledge and co-workers. ${ }^{1,38-43}$ The IMC method generates configurations consisting of a noncrystalline domain bounded by two static crystalline domains, which are semi-infinite in their lateral dimensions due to periodic boundary conditions. The resulting ensemble is metastable: the crystalline domain boundaries prevent complete melting, while the reduced density prevents complete crystallization. In these configurations, the number-average molecular weight can be related statistically to the size of the simulation and the number of chain ends introduced. ${ }^{44}$ Local displacement moves as well as chain connectivity-altering Monte Carlo moves were performed in the noncrystalline domain to ensure sampling of the packing and topological spaces. The IMC method has been used with some success to calculate a variety of thermomechanical properties of SCPE, to characterize interfacial stresses and chain tilt within the crystal lamellae, and, more recently, to generate initial configurations for large strain deformations of SCPE. For further details regarding the IMC methodology, the reader is referred to the original works. ${ }^{1,38-43}$

The IMC method generates SCPE configurations in a simplified description where methylene $\left(\mathrm{CH}_{2}\right)$ and methyl $\left(\mathrm{CH}_{3}\right)$ groups are treated as lumped sites in a so-called united atom (UA) model. The details of the IMC procedure for generation of SCPE configurations are discussed below. First, an orthorhombic crystal lattice of dimensions $3 \times 5 \times 50$ unit cells was created with a total of 3000 UA sites. The lattice parameters of the PE unit cell were $[a=0.772 \mathrm{~nm}, b=0.444$ $\mathrm{nm}, c=0.253 \mathrm{~nm}] .^{45}$ The crystal stems in the lattice were oriented with the $\{201\}$ crystallographic plane normal to the slab longitudinal $(z)$ direction, which corresponds to the most energetically favorable crystal-amorphous interface. ${ }^{41,46}$ The simulation box was necessarily monoclinic to satisfy periodic boundary conditions ${ }^{1}$ with box dimensions $\left[L_{x}=2.767 \mathrm{~nm}, L_{y}\right.$ $\left.=2.223 \mathrm{~nm}, L_{z}=10.57 \mathrm{~nm}\right]$ and angles $\left[\alpha=90^{\circ}, \beta=97.51^{\circ}, \gamma\right.$ $\left.=90^{\circ}\right] .1500$ sites in the middle of the box were subject to amorphization through MC simulation. The remaining 1500 sites were ignored in the MC simulation and held static in the crystal lattice structure. A total of 264 sites were removed from six randomly selected chains in the noncrystalline region to set its density to approximately $0.85 \mathrm{~g} / \mathrm{cm}^{3}$. Thus, the initial crystallinity of the configurations for subsequent $\mathrm{MD}$ simulations was about 55 mass \%, and the number-average molecular weight was approximately $6400 \mathrm{~g} / \mathrm{mol}$. The noncrystalline domain of the slab underwent amorphization at $10000 \mathrm{~K}$ for $120000 \mathrm{MC}$ cycles, followed by a quench step to $350 \mathrm{~K}$ over $40000 \mathrm{MC}$ cycles in the NVT ensemble. The distribution of bond lengths, angles, and torsions, which are most critical in the vibrational analysis performed here, were inspected to ensure thermal equilibration at $350 \mathrm{~K}$ during the quench step. A possible issue with a single quench step is the incomplete equilibration of topological structures at this lower temperature. Previous studies have overcome this issue by using a stepwise quenching procedure. ${ }^{1,47}$ We confirmed that the vibrational spectrum did not change significantly when calculated from configurations using a stepwise quenching procedure as compared to the single quench step used in this work (see Supporting Information for details). Each MC cycle consisted of $N_{\text {mobile }}$ MC moves where $N_{\text {mobile }}$ is the number of mobile sites. Ten configurations were sampled at equal intervals from a $100000 \mathrm{MC}$ cycles run at $350 \mathrm{~K}$ in the NVT ensemble. The set of MC moves and frequencies as described in Lee and Rutledge $^{43}$ were used here; Yeh et al. ${ }^{47}$ found that the model's topological features were not particularly sensitive to different frequencies of moves in long MC runs. After equilibration using the IMC method, ten representative configurations of SCPE thus generated were ported to LAMMPS $^{48}$ for complete thermalization of amorphous, crystalline, and interphase domains, as described later.

In this work, the valence and nonbonded interactions of the UA sites were governed by the force field originally developed by Paul et al., ${ }^{49}$ with the modifications proposed by Bolton et al. ${ }^{50}$ As typical for PE descriptions lacking explicit hydrogens, this UA model does not predict the orthorhombic crystal phase at the conditions in this work. Instead, upon thermalization in MD the crystal phase captured by the UA description resembled a hexagonal rotator phase in which crystal stems have no preferred setting angle. ${ }^{51}$ In past works, the UA force field has been shown to predict the melting points and heats of fusion for rotator phases of $n$-alkanes in good agreement with experimental findings. ${ }^{52}$

It should be noted that only the skeletal vibrations, where the $\mathrm{CH}_{2}$ moiety moves as a single site, can be analyzed in the UA description. An explicit atom (EA) model is required to examine vibrations involving $\mathrm{C}-\mathrm{H}$ interactions. Furthermore, EA force fields for PE are capable of reproducing both the orthorhombic and rotator crystal structures, ${ }^{51}$ which were suggested to be important in producing the crystalline bending doublet, key to the observation of a distinct interphase spectrum. ${ }^{6}$ For these reasons, a simple reverse-mapping procedure was developed to introduce hydrogen atoms into the UA configurations, using the geometry and connectivity of the UA sites. Positions of the hydrogens within the local frame of a $\mathrm{CH}_{2}$ site are given by

$$
\begin{aligned}
& \mathbf{r}_{\mathrm{H} 1}=\mathbf{r}_{i}-d_{0} \sin \left(\frac{\theta_{0}}{2}\right) \mathbf{v}_{3}-d_{0} \cos \left(\frac{\theta_{0}}{2}\right) \mathbf{v}_{2} \\
& \mathbf{r}_{\mathrm{H} 2}=\mathbf{r}_{i}+d_{0} \sin \left(\frac{\theta_{0}}{2}\right) \mathbf{v}_{3}-d_{0} \cos \left(\frac{\theta_{0}}{2}\right) \mathbf{v}_{2}
\end{aligned}
$$

where $\mathbf{v}_{1}, \mathbf{v}_{2}$, and $\mathbf{v}_{3}$ are local coordinate vectors of the UA site as illustrated in Figure $2 \mathrm{a} ; \mathbf{r}_{\mathrm{H} 1}, \mathbf{r}_{\mathrm{H} 2}$, and $\mathbf{r}_{i}$ are the Cartesian position vectors for the two methylene hydrogens and site $i$, respectively; $d_{0}$ is the equilibrium distance for the $\mathrm{C}-\mathrm{H}$ bond, and $\theta_{0}$ is the equilibrium value for the $\mathrm{H}-\mathrm{C}-\mathrm{H}$ angle. Figure $2 \mathrm{~b}$ depicts the inserted methylene hydrogens in the local coordinate frame of site $i$. To specify fully the positions of the methyl hydrogens, one of the $\mathrm{H}-\mathrm{C}-\mathrm{C}-\mathrm{C}$ dihedrals was set to be in the trans position.

Using the reverse-mapping procedure, EA configurations were generated from the ten UA configurations described above. For describing valence and nonbonded interactions in the EA description, the COMPASS force field developed by Sun $^{53}$ was used. This force field has been shown to reproduce molecular vibration frequencies in good agreement with experimental data for $n$-alkanes. ${ }^{53}$ Additionally, the structural, conformational, and thermodynamic properties of the ortho- 
(a)

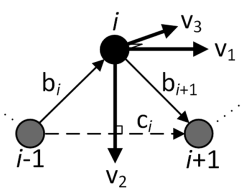

(b)

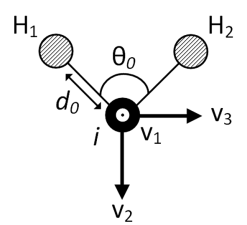

Figure 2. (a) Schematic of the local coordinate frame of site $i$ with base vectors of $\mathbf{v}_{1}, \mathbf{v}_{2}$, and $\mathbf{v}_{3}$ for the UA description of PE. Vector $\mathbf{v}_{1}$ has an origin at site $i$ and is parallel to the bond chord vector $\mathbf{c}_{i}$. Vector $\mathbf{v}_{2}$ is perpendicular to $\mathbf{v}_{1}$ and bisects the angle between bond vectors $\mathbf{b}_{i}$ and $\mathbf{b}_{i+1}$. To complete the right-handed coordinate frame, $\mathbf{v}_{3}$ is defined as the cross product $\mathbf{v}_{3}=\mathbf{v}_{1} \times \mathbf{v}_{2}$ and is normal to the plane of bond vectors $\mathbf{b}_{i}$ and $\mathbf{b}_{i+1}$. (b) Illustration of the inserted methylene hydrogen atoms for site $i$ along with its local coordinate frame. The figure is oriented such that $\mathbf{v}_{1}$ is coming out of the page.

rhombic PE crystal phase as well as its infrared vibrational spectrum have been previously studied through atomistic MD simulations using the COMPASS force field with satisfactory accuracy; ${ }^{28}$ the computed Raman spectrum for crystalline PE was not reported.

2.2. Molecular Dynamics Simulation Details. All MD simulations and energy minimizations in this work were performed using the LAMMPS ${ }^{48}$ software package. For all simulations, a velocity-Verlet scheme ${ }^{54}$ was used to integrate Newton's equations of motion with a time step of $1 \mathrm{fs}$ (unless specified otherwise), and constant temperature was maintained by a Nosé-Hoover thermostat with a damping constant of 100 time steps. For simulations in the NPT ensemble, constant pressure was maintained using a Nosé-Hoover barostat with a damping constant of 1000 time steps, and the diagonal components of the pressure tensor were controlled independently. The output configurations generated using the IMC methodology (with an additional reverse-mapping step for the EA configurations) underwent an energy minimization, followed by a short, 100 ps relaxation in the NVT ensemble at $100 \mathrm{~K}$. Next, the structures were equilibrated at constant stress conditions for $1 \mathrm{~ns}$ in the NPT ensemble at $100 \mathrm{~K}$ and 1 atm. Following equilibration, production simulations of $100 \mathrm{ps}$ were performed in the NVT ensemble at $100 \mathrm{~K}$ with a time step of $0.1 \mathrm{fs}$. The nonbonded interactions were captured using a Lennard-Jones potential in the UA description and a 9-6 potential in the EA description with a radial cutoff distance of $2.5 \sigma$, where $\sigma=0.4 \mathrm{~nm}$ is the van der Waals diameter of the UA $\mathrm{CH}_{2}$ site. Long-range van der Waals corrections to energy and pressure were included in these simulations. ${ }^{53}$ For simulations in the EA description, long-range Coulombic interactions were computed using a particle-particle particle-mesh solver with a relative force accuracy of 0.001 . The Eckart conditions ${ }^{55}$ were enforced during the simulation by zeroing the linear and angular momentum of the entire system every 10 time steps through adjustment of the site velocities.

2.3. Vibrational Analysis Procedure. The vibrational analysis used in this work was developed based upon the methodology proposed by Strachan. ${ }^{14}$ In this approach, the vibrational spectrum $P(v)$ is calculated within the quasiharmonic approximation ${ }^{16}$ from the power density of the massweighted velocities, given by

$$
P(v)=2 \beta \sum_{j=1}^{3 N} \lim _{\tau \rightarrow \infty} \frac{1}{2}\left|\int_{-\tau}^{\tau} \mathrm{d} t \exp (-i 2 \pi v t) v_{j}^{\prime}(t)\right|^{2}
$$

where $v_{j}^{\prime}(t)=\sqrt{m_{j}} v_{j}(t) / 2$ is the $j$ th component of the $3 N$ massweighted Cartesian velocity vector, $N$ is the number of sites, and $\beta=1 / k T$ is the normalization factor:

$$
\beta^{-1}=\frac{2}{3 N} \lim _{\tau \rightarrow \infty} \int_{-\tau}^{\tau} \mathrm{d} t \frac{1}{2 \tau} \sum_{j=1}^{3 N} v^{\prime}{ }_{j}(t)^{2}
$$

Briefly, Strachan's method allows for the decomposition of the power density spectrum given by eq 2 into contributions from $3 N$ NM's, using the $3 N \times 3 N$ mass-weighted covariance matrix of $x$ :

$$
K_{i j}^{x}=\left\langle x_{i}^{\prime} x_{j}^{\prime}\right\rangle / 2 \quad \text { for } i, j=1 \ldots 3 N
$$

where $x^{\prime}$ is the mass-weighted Cartesian position $r^{\prime}$, velocity $v^{\prime}$, or acceleration $a^{\prime}$. The $\langle\ldots\rangle$ indicates a time average over the duration of the simulation. In this work, we used the massweighted acceleration covariance matrix $\mathbf{K}^{a}$. This choice was motivated by the observation of NM spectra with better resolved peaks when given by the eigenvectors of $\mathbf{K}^{a}$ as compared to those of $\mathbf{K}^{v}$, in preliminary calculations on a small PE crystal.

To distinguish vibrations from liquidlike motions, the massweighted Cartesian accelerations were projected into a local reference frame before computing $\mathbf{K}^{a}$ and $P(v)$, according to

$$
\hat{a}_{n}(t)=\sum_{i=1}^{3 N} B_{n, i}(t) \cdot a_{i}^{\prime}(t)
$$

where $\hat{a}_{n}$ is the mass-weighted acceleration projected into the local vector $n$. The $3 N \times 3 N$ matrix $\mathbf{B}(t)$, with elements $B_{n, i}$ serves to transform accelerations from the Cartesian frame to those in a local frame. The local reference frame utilized for the UA description, depicted in Figure 2a, is adapted from Mavrantzas and Theodorou. ${ }^{56}$ This local frame was selected because it conveniently coincides with the theoretical skeletal vibrations of an infinite PE chain ${ }^{26}$ where $\mathbf{v}_{1}, \mathbf{v}_{2}$, and $\mathbf{v}_{3}$ correspond to the stretching, bending, and twisting directions of the $i$ th site. The local frame for carbon atoms in the EA description was identical to the local frame used for the united atom UA description. Additionally, a similar frame was devised for the hydrogen atoms in the EA description. Further details of the local reference frame are provided in the Supporting Information. In addition to removing liquidlike motions, local reference frames are more convenient for the analysis of vibrational NM's than the Cartesian coordinate. ${ }^{13}$

Following projection into a local reference frame, the vibrational spectrum was then decomposed into contributions from $3 N$ local NM's, using the $3 N \times 3 N$ local acceleration covariance matrix, computed by

$$
\hat{K}_{i j}^{a}=\left\langle\hat{a}_{i} \hat{a}_{j}\right\rangle / 2 \quad \text { for } i, j=1 \ldots 3 N
$$

Next, $\hat{\mathbf{K}}^{a}$ is diagonalized into its $3 N$ eigenvectors $\xi_{k}$ and eigenvalues $\lambda_{k}$, of which only $3 N-6$ are nonzero. The eigenvectors corresponding to the nonzero eigenvalues, which describe the atomic displacement within each NM, were used to assign the NM's to vibration types. The local accelerations were then transformed into modal accelerations $\tilde{a}_{k}(t)$ by 


$$
\tilde{a}_{k}(t)=\sum_{n=1}^{T} \xi_{k, n} \hat{a}_{n}(t)
$$

such that the modal accelerations were uncorrelated:

$$
\left\langle\hat{a}_{l}(t) \hat{a}_{m}(t)\right\rangle \propto \delta_{l m}
$$

where $\delta_{l m}$ is Kronecker's delta. The spectrum of each NM, $P_{k}(v)$, was given by the power density spectrum of $\tilde{a}_{k}(t)$ :

$$
P_{k}(v)=\lim _{\tau \rightarrow \infty} \frac{1}{2 \tau}\left|\int_{-\tau}^{\tau} \mathrm{d} t \exp (-i 2 \pi v t) \tilde{a}_{k}(t)\right|^{2}
$$

The modal accelerations were analyzed over the 100 ps simulation with a sampling time of 1 fs. Under these parameters, the maximum resolved frequency was 16667 $\mathrm{cm}^{-1}$ with a spectral resolution of $0.33 \mathrm{~cm}^{-1}$. The overall vibrational spectrum was obtained by summing the contributions from the $3 N-6$ modes, obtained using eq 9 .

In addition to analyzing the vibrational spectrum of the entire SCPE model, it is also possible to restrict the calculation of vibrational spectra to sites within the distinct domains of the semicrystalline slab (i.e., crystalline, interphase, and the amorphous). To accomplish this, a methodology for the assignment of sites to domains was developed. The local bond orientation parameter for each site, given by

$$
\left\langle P_{2, i}\right\rangle=\frac{3}{2}\left\langle\cos ^{2} \theta_{i j}\right\rangle-\frac{1}{2}
$$

was used to label a site as either crystalline, interphase, or amorphous, where $\theta_{i j}$ is the angle between the vector from the site $(i-1)$ to the site $(i+1)$ and the vector from site $(j-1)$ to the site $(j+1)$. The $\langle\ldots\rangle$ indicates an average over all sites within a cutoff radius $r_{\mathrm{c}}$ from the site $i$ (hence the term "local") and over all frames considered in the analysis. In the EA description, only the carbon atoms were considered as sites, with the hydrogen atoms sharing the same assignment as the carbon to which they were bonded. $\left\langle P_{2, i}\right\rangle$ was computed from 1000 frames, collected at intervals of $100 \mathrm{fs}$ during the $100 \mathrm{ps}$ simulation. Motivated by the work of Rutledge and coworkers, ${ }^{1,52}$ the cutoff radius $r_{\mathrm{c}}=2.5 \sigma$ was used for calculating $\left\langle P_{2, i}\right\rangle$ in this work. Each site was assigned to a domain based on the "90-10" rule, ${ }^{57}$ where $\left\langle P_{2, i}\right\rangle>0.9$ indicates a crystalline site, $\left\langle P_{2, i}\right\rangle<0.1$ indicates an amorphous site, and everything in between indicates an interphase site. These selection criteria mirror the qualitative definitions of the crystalline, interphase, and amorphous domains as being high, intermediate, and low order, respectively. It should be noted that the assigned domain of each site was found to be invariant to the number of frames used to compute $\left\langle P_{2, i}\right\rangle$; identical assignments were obtained using 100,1000, and 100000 equally spaced frames over the run duration. The NM's and $P(v)$ for each domain were calculated by applying Strachan's method selectively to sites within a domain.

\section{RESULTS AND DISCUSSION}

3.1. Results from United Atom Model. Following the equilibration procedure discussed above, the average dimensions ( \pm 1 standard deviation) of the simulation box used for the UA vibrational analysis runs were $\left[L_{x}=2.774 \pm 0.02 \mathrm{~nm}, L_{y}\right.$ $\left.=2.278 \pm 0.01 \mathrm{~nm}, L_{z}=10.44 \pm 0.04 \mathrm{~nm}\right]$. The lateral dimensions of the box expanded slightly upon thermal equilibration of the crystalline layers, while the longitudinal dimension contracted, relative to the box dimensions employed in the Monte Carlo simulations, for a net increase in volume of $1.4 \%$.

Figure 3 shows the spatial variation of $\left\langle P_{2, i}\right\rangle$ in the longitudinal $(z)$ direction of the slab, denoted here as

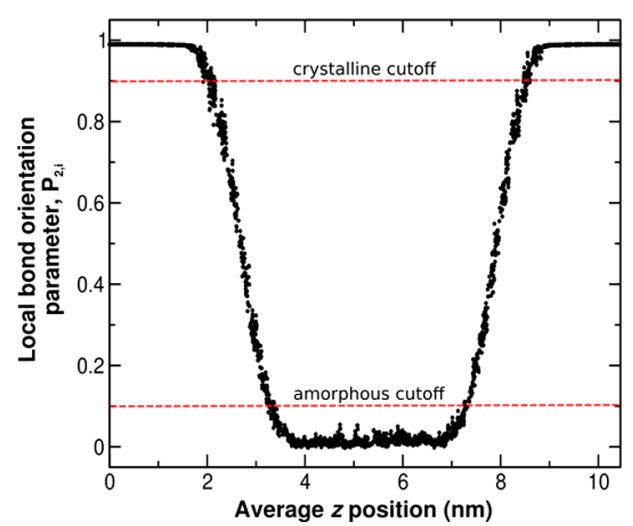

Figure 3. Profile of the local bond orientation parameter in which the $\left\langle P_{2, i}\right\rangle$ of each site is shown against its average $z$ position during the vibrational analysis run (filled circles) for a typical UA configuration. The crystalline and amorphous cutoff values are indicated by the red dashed lines.

$\left\langle P_{2, i}(z)\right\rangle$, for a typical UA configuration. The $\left\langle P_{2, i}(z)\right\rangle$ profile is nearly symmetric about the slab midplane and smoothly decreases from the crystalline region to amorphous region. The crystalline domains located at each end of the box retained their high degree of order during the run $\left(\overline{\left\langle P_{2, i}^{\text {cry }}\right\rangle}=0.983\right)$, while the amorphous domain located in the middle of the slab was disordered $\left(\overline{\left\langle P_{2, i}^{\text {amorph }}\right\rangle}=0.020\right)$. Clear demarcation boundaries perpendicular to the $z$ direction separate the domains, which is consistent with the conceptualized lamellar stack as illustrated in Figure 1. From $\left\langle P_{2, i}(z)\right\rangle$, the width of the interphase region is estimated to be $1.22 \pm 0.06 \mathrm{~nm}$, which agrees favorably with previous simulation estimates ${ }^{38,39,41}$ and values based on SAXS and Raman measurements. ${ }^{6,58}$ These observations reinforce the parameter choices for cutoff radius and selection criteria used for the assignment of sites to domains. A typical UA configuration generated via the IMC methodology and subsequently equilibrated is depicted in Figure 4. The crystalline, interphase, and amorphous sites are colored in red, green, and blue, respectively. On average, the configurations for the UA model consist of $1144 \pm 13$ crystalline sites (42\%), $652 \pm 11$ interphase sites (24\%), and $939 \pm 7$ amorphous sites (34\%).

Figure 5 shows the overall vibrational spectrum for SCPE using the UA model, averaged over the ten configurations. Formally, this spectrum captures the average spectral density of the total inverse mass-weighted squared force acting on all sites in the slab (i.e., $\sum_{i}\left\langle F_{i}^{2}\right\rangle / m_{i}$ ). The spectrum is normalized such that the highest peak has unit intensity. The vibrations captured by the UA model correspond to carbon skeletal modes. For theoretical details of the skeletal modes for carbon chain molecules, the reader is referred to the first column of diagrams in Figure 9 of Krimm et al. ${ }^{26}$ Sharp peaks occur in the total spectrum at approximately 1440, 1193, 1147, 480, and 230 $\mathrm{cm}^{-1}$. The peaks were assigned based on the eigenvectors of $\mathrm{K}^{a}$. Based on their eigenvectors, the peaks at 1193 and $1440 \mathrm{~cm}^{-1}$ were assigned respectively to the skeletal symmetric $\mathrm{C}-\mathrm{C}$ bond stretch and the skeletal asymmetric $\mathrm{C}-\mathrm{C}$ bond stretch modes. 


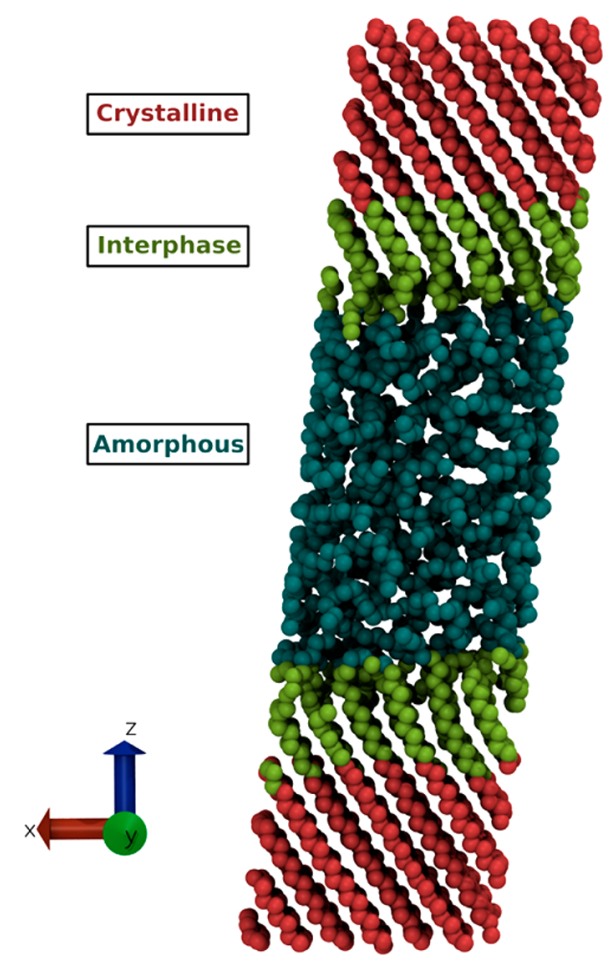

Figure 4. A typical SCPE configuration for the UA model rendered in VMD. ${ }^{59}$ The crystalline, interphase, and amorphous sites are colored in red, green, and blue, respectively. The Cartesian directions are illustrated in the lower left corner.

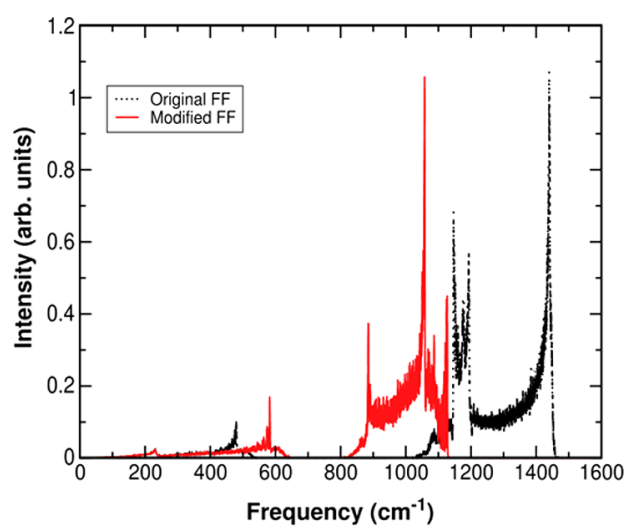

Figure 5. Comparison of the overall vibrational spectra for SCPE using the original UA force field and the modified UA force field with different stretching and bending force constants, $\hat{k}_{\mathrm{s}}$ and $\hat{k}_{\mathrm{b}}$ (averaged over ten configurations). The standard error associated with each spectrum is captured by the line width. The spectra were normalized such that the maximum peak has unit intensity.

The UA force field used in this work overestimates the vibrational frequencies of the skeletal symmetric and asymmetric $\mathrm{C}-\mathrm{C}$ stretching modes, which have observed frequencies of 1126 and $1059 \mathrm{~cm}^{-1}$, respectively. ${ }^{27,32}$ To further investigate this observation, the skeletal vibrational frequencies were estimated from the valence force field parameters using the procedure of Kirkwood ${ }^{60}$ as later modified by Liang et al. ${ }^{6}$ Briefly, this approach estimates the skeletal frequencies of an all-trans chain of $\mathrm{CH}_{2}$ point masses using the stretching force constant $k_{\mathrm{s}}$, bending force constant $k_{\mathrm{b}}$, equilibrium bond distance, and equilibrium angle. Using the values from the UA force field, the skeletal symmetric and asymmetric CC stretch frequencies are estimated to be 1164 and $1443 \mathrm{~cm}^{-1}$, respectively. The estimated frequencies agree well with those calculated via simulation. Since the UA force field used in this work was not originally parametrized to reproduce vibrational frequencies, it is not surprising that the force field does not reproduce vibrational frequencies in good agreement with experimental values. To recover the experimental skeletal frequencies using Kirkwood's procedure, $k_{\mathrm{s}}$ should be decreased from $3.76 \times 10^{5}$ to $\left.2.02 \times 10^{5} \mathrm{~kJ} /(\mathrm{mol} \mathrm{nm})^{2}\right)$ and $k_{b}$ increased from 500 to $929 \mathrm{~kJ} /\left(\mathrm{mol} \mathrm{rad}^{2}\right)$.

The overall vibrational spectrum was recalculated using the modified UA force field with the estimates of $k_{\mathrm{s}}$ and $k_{\mathrm{b}}$ from Kirkwood's procedure, denoted as of $\hat{k}_{\mathrm{s}}$ and $\hat{k}_{\mathrm{b}}$. Interestingly, $\hat{k}_{\mathrm{s}}$ reproduces the frequency of the asymmetric $\mathrm{C}-\mathrm{C}$ stretching mode, but $\hat{k}_{\mathrm{b}}$ overestimates the frequency of the symmetric $\mathrm{C}-$ $\mathrm{C}$ stretching mode by approximately $47 \mathrm{~cm}^{-1}$. Through linear interpolation of the two frequencies, a new $\hat{k}_{\mathrm{b}}=828 \mathrm{~kJ} /(\mathrm{mol}$ $\left.\operatorname{rad}^{2}\right)$ is estimated to recover the observed frequency of the symmetric stretching mode. Figure 5 shows the overall vibrational spectrum for SCPE using the modified UA force field as compared to the previous force field. The modified UA force field reproduces the vibrational frequencies of the symmetric and asymmetric $\mathrm{C}-\mathrm{C}$ stretching modes, 1125 and $1058 \mathrm{~cm}^{-1}$, respectively, in good agreement with the observed frequencies. In the modified UA force field, the torsional potential is unchanged, indicated by the unaltered peak of torsional mode located at $230 \mathrm{~cm}^{-1}$. Although these revised force constants are recommended for future work, the remainder of the analysis presented here applies to the original UA force field.

Next, the vibrational spectrum of each domain was calculated individually. Figure 6a illustrates the vibrational spectra for the three domain types, averaged over the ten UA configurations. The sharp peaks observed at $1193,1147,480$, and $230 \mathrm{~cm}^{-1}$ in the overall vibrational spectrum can be attributed to the crystalline domain, while the peaks at 1174 and $1122 \mathrm{~cm}^{-1}$ can be attributed to the amorphous domain. The spectrum of the interphase domain is observed to contain peaks from both crystalline and amorphous domains. Table 1 presents a summary of the vibrational peaks and comparison to observed frequencies, where experimental data exist. Table 1 demonstrates that the frequencies of certain modes are shifted between the domains. Most notably, the frequencies of the symmetric stretching modes in the crystalline domain are downshifted in the amorphous domain, as depicted by Figure 6b. This shift is thought to be a result of chain confinement in the lateral dimension by the lattice, which leads to bending modes with higher frequencies in the crystalline domain. Furthermore, the frequency of the aymmetric stretching mode was found to be nearly invariant between the domains, suggesting this mode is insensitive to local environment. Additionally, peaks arising due to the coupling between the skeletal stretching modes and torsional modes are observed in the UA model.

The vibrational results of the UA model demonstrate that the proposed method of vibrational analysis is a viable approach to calculate the spectra for the domains of SCPE and allows to assign the spectral peaks using the eigenvector of $\mathbf{K}^{a}$. Additionally, the UA results indicate the importance of explicit hydrogen atoms in adequately characterizing the vibrational spectra of SCPE and its domains. Hence, an investigation of the vibrational spectra of SCPE in an EA description follows next. 

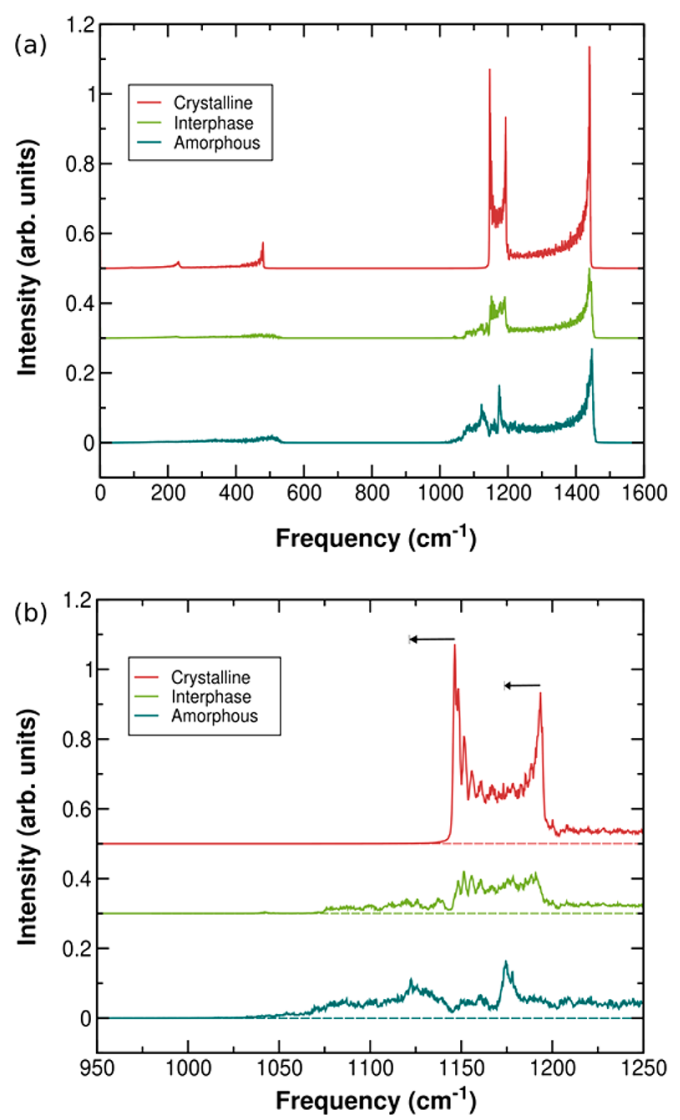

Figure 6. (a) Vibrational spectra for the crystalline, interphase, and amorphous domains of SCPE in the UA model, averaged over the ten configurations. (b) Expanded view of (a) where arrows indicate the shift in the frequencies between the crystalline to amorphous domain. For clarity, the interphase and crystalline spectra are shifted in intensity by adding 0.3 and 0.5 , respectively. The standard error associated with each spectrum is shown by its line width. The spectra were normalized to the maximum peak in the total vibrational spectrum.

Table 1. Wavenumber of the Vibrational Peaks in the Spectra of the Domains in SCPE for the UA Model at $T=$ $100 \mathrm{~K}$

\begin{tabular}{clc}
$\begin{array}{c}\text { present work } \\
\left(\mathrm{cm}^{-1}\right)\end{array}$ & \multicolumn{1}{c}{ skeletal mode description } & obsd Raman $\left(\mathrm{cm}^{-1}\right)$ \\
1440 & asym stretch, crystal + amorph & $1059(\mathrm{~m})^{27}$ \\
1193 & sym stretch, crystal & $1126(\mathrm{~m})^{27}$ \\
1174 & sym stretch, amorph & $1080^{32}$ \\
1147 & sym + asym stretch, crystal & \\
1122 & sym + asym stretch, amorph & \\
480 & torsion + sym stretch, crystal & \\
230 & torsion, crystal \\
\hline
\end{tabular}

3.2. Results from Explicit Atom Model. Following reverse mapping of the UA configurations to include hydrogens and thermal equilibration with the EA force field, the simulation box for the EA model contracted slightly, with average dimensions of $\left[L_{x}=2.654 \pm 0.062 \mathrm{~nm}, L_{y}=2.372 \pm 0.074\right.$ $\left.\mathrm{nm}, L_{z}=10.53 \pm 0.04 \mathrm{~nm}\right]$. Based on these dimensions and the tilt angle of the chain stems in the crystalline domain relative to the $z$-axis, the unit cell parameters were estimated to be $a=$ $0.713 \mathrm{~nm}$ and $b=0.474 \mathrm{~nm}$ at $100 \mathrm{~K}$; these values are in good agreement with experimental values at $103.2 \mathrm{~K}$ reported by
Davis et al. $^{62}$ The ratio $a / b=1.504$ is consistent with the orthorhombic structure. Figure 7 shows the distribution of the

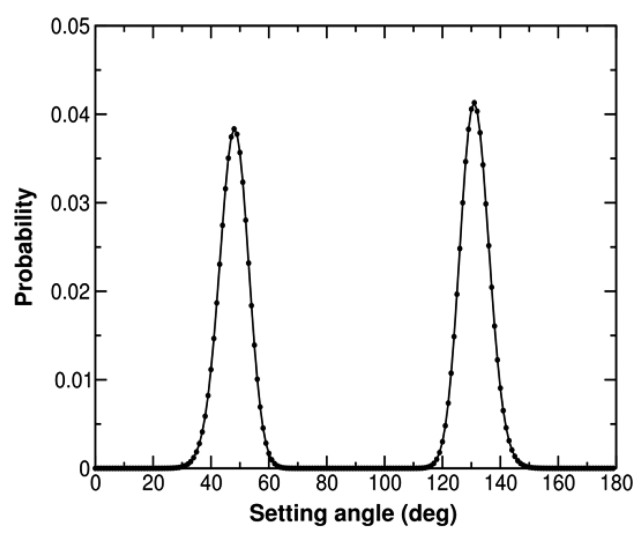

Figure 7. Distribution of the local setting angle in the crystalline domain for the EA model, averaged over the ten configurations.

local setting angles in the crystalline domain, as calculated according to Zubova et al. ${ }^{63}$ The local setting angle is defined by the azimuthal angle of a $\mathrm{C}-\mathrm{C}-\mathrm{C}$ angle bisector vector in the $x y$ plane, with a value between $[0,2 \pi]$. The distribution of setting angle in the crystalline domain shows two peaks at $48^{\circ}$ and $132^{\circ}$, in good agreement with experiments ${ }^{64}$ and simulations of orthorhombic PE crystals. ${ }^{28,51}$ The typical "herringbone" chain arrangement associated with the orthorhombic crystal phase has alternating rows of chains with setting angles of $\psi$ and $(180-\psi)$ and was confirmed by visual inspection.

The assignment of sites in the EA model used the same cutoff radius and values for domain selection as the UA model analysis. Figure 8 depicts $\left\langle P_{2, i}(z)\right\rangle$ for a typical EA

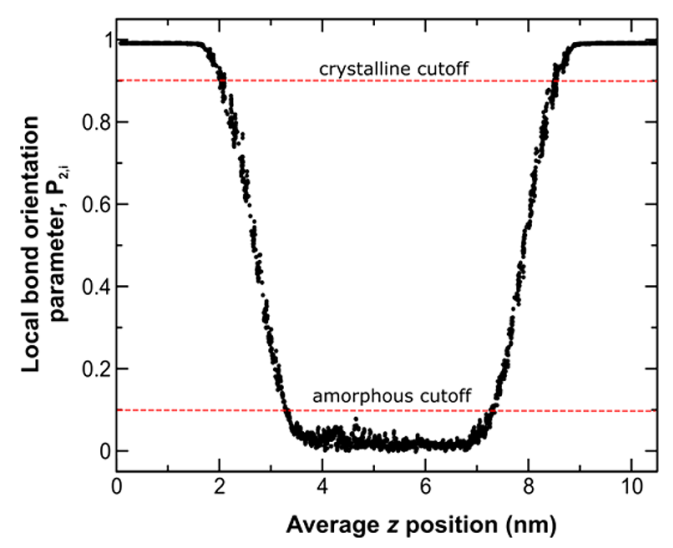

Figure 8. Profile of the local $\mathrm{C}-\mathrm{C}$ bond orientation parameter in which the average $\left\langle P_{2, i}\right\rangle$ for each carbon site is shown against its average $z$ position during the vibrational analysis run (filled circles) for a typical EA configuration. The crystalline and amorphous cutoff values are indicated by the red dashed lines.

configuration. The order in the crystalline and amorphous domains of the EA model is comparable to the UA model, with $\overline{\left\langle P_{2, i}^{\text {cry }}\right\rangle}=0.987$ and $\overline{\left\langle P_{2, i}^{\text {amorph }}\right\rangle}=0.022$. Several sites with unusually high order in the amorphous region are observed between $z=4.0 \mathrm{~nm}$ and $z=5.5 \mathrm{~nm}$, with the $\left\langle P_{2, i}\right\rangle$ of some sites close to the amorphous cutoff value. The width of the interphase region in the EA model was slightly greater than the 
UA model with an estimate of $1.30 \pm 0.08 \mathrm{~nm}$ but still in the range of values from SAXS and Raman measurements. ${ }^{6,58}$ The average EA configuration consists of $3424 \pm 40$ crystalline atoms (42\%), $1989 \pm 58$ interphase sites (24\%), and $2807 \pm 24$ amorphous sites (34\%). The phase composition for the EA configurations is similar to the UA configurations, providing support for the choice of parameters in the assignment of EA sites.

Figure 9 shows the overall vibrational spectrum for SCPE in the EA model, averaged over the ten configurations, while the

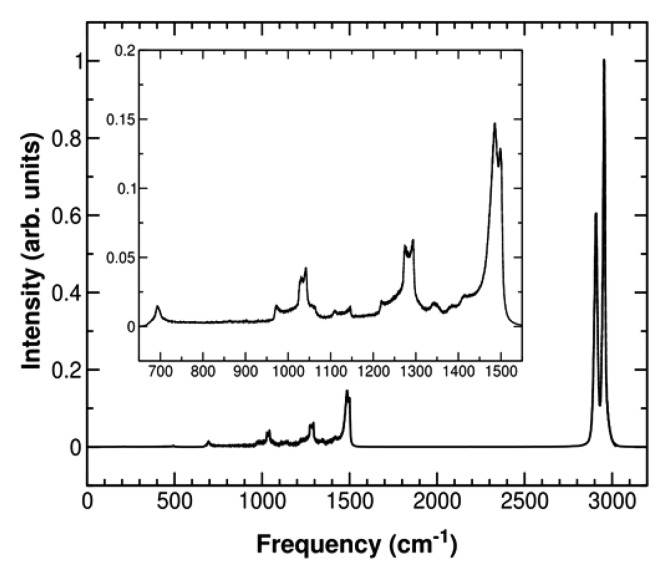

Figure 9. Overall vibrational spectrum for SCPE in the EA model averaged over the ten configurations. The inset illustrates an expanded view of the overall spectrum in the region between 650 and 1550 $\mathrm{cm}^{-1}$. The standard error associated with the spectrum is captured by the line width. The spectrum was normalized such that the maximum peak has unit intensity.

inset highlights the region from 650 to $1550 \mathrm{~cm}^{-1}$ in an expanded view. Whereas the UA model captured only skeletal vibrational modes, the inclusion of explicit hydrogens in the EA model produces vibrational modes internal to the $\mathrm{CH}_{2}$ group. The overall spectrum in the EA model is in qualitative agreement with typical infrared and Raman signatures of alkanes $^{65}$ and low-density PE. ${ }^{32}$ The high-frequency doublet near $3000 \mathrm{~cm}^{-1}$ is characteristic of $\mathrm{CH}_{2}$ stretching vibrations. A $\mathrm{CH}_{2}$ bending band is seen between 1400 and $1500 \mathrm{~cm}^{-1}$ as well as $\mathrm{CH}_{2}$ twisting/wagging band near $1300 \mathrm{~cm}^{-1}$. Based on comparison to the infrared spectrum of $\mathrm{PE}$ calculated by Mavrantza et al. ${ }^{28}$ using the same force field, the peak at 1500 $\mathrm{cm}^{-1}$ is determined to be infrared-active, while the peak at 1484 $\mathrm{cm}^{-1}$, not observed by them, is determined to be Raman-active. Lastly, a low-frequency peak near $700 \mathrm{~cm}^{-1}$ is observed, which is typical of the $\mathrm{CH}_{2}$ rocking mode. The modes of these major peaks were confirmed using eigenvector information.

Table 2 compares the calculated peaks in the overall vibrational spectrum of SCPE in the EA model to experimental observations. A few discrepancies are observed between the calculated spectrum and experimental data. First, despite confirming an orthorhombic structure in the crystalline domain (see Figure 7 and associated discussion), the EA model does not predict the peak associated with the orthorhombic crystal phase near $1416 \mathrm{~cm}^{-1}$. With the exception of the $\mathrm{CH}_{2}$ rocking mode, the $\mathrm{CH}$ vibrations tend to be slightly upshifted relative to experimental observations, but generally within $\pm 50 \mathrm{~cm}^{-1}$ of observed frequencies. Some of this discrepancy may be explained by the tendency of peaks to narrow and shift upward in frequency with decreasing temperature, by about $0.01-0.05$
Table 2. Wavenumber of the Vibrational Peaks in the Total Spectrum of SCPE in the EA Model at $T=100 \mathrm{~K}$

$\begin{array}{llll}\begin{array}{c}\text { present } \\ \text { work }\left(\mathrm{cm}^{-1}\right)\end{array} & \text { mode description }{ }^{29} & \begin{array}{c}\text { obsd }^{27} \text { Raman } \\ \left(\mathrm{cm}^{-1}\right)\end{array} & \begin{array}{c}\text { obsd }^{27} \text { infrared } \\ \left(\mathrm{cm}^{-1}\right)\end{array} \\ 2954 & \mathrm{CH}_{2} \text { asym stretch } & 2883(\mathrm{~s}) & 2920(\mathrm{vs}) \\ 2906 & \mathrm{CH}_{2} \text { sym stretch } & 2848(\mathrm{~s}) & 2848(\mathrm{vs}) \\ 1500 & \mathrm{CH}_{2} \text { bend } & & 1473,1463(\mathrm{~s}) \\ 1484 & \mathrm{CH}_{2} \text { bend } & 1437,1413(\mathrm{~m}) & \\ 1293 / 1276 & \mathrm{CH}_{2} \text { twist } & 1292(\mathrm{~m}) & \\ 1150 & \text { sym C-C stretch } & 1126(\mathrm{~m}) & \\ 1042 & \text { asym C-C stretch } & 1059(\mathrm{~m}) & \\ 691 & \mathrm{CH}_{2} \text { rock } & & 731,720(\mathrm{~s})\end{array}$

$\mathrm{cm}^{-1} / \mathrm{K}$ (see Supporting Information). Somewhat better agreement with the experimentally observed infrared frequencies was reported by Mavrantza et al. using the COMPASS force field and a normal-mode analysis of the Hessian matrix. ${ }^{28}$ Nevertheless, discrepancies in peak positions of $25-50 \mathrm{~cm}^{-1}$ are not uncommon in calculations using empirical force fields. ${ }^{29}$

Figure 10a illustrates the entire vibrational spectra for the three domains in the EA model averaged over the ten configurations. The vibrational peaks between 700 and 1500 $\mathrm{cm}^{-1}$ are shown more clearly in the expanded spectrum Figure
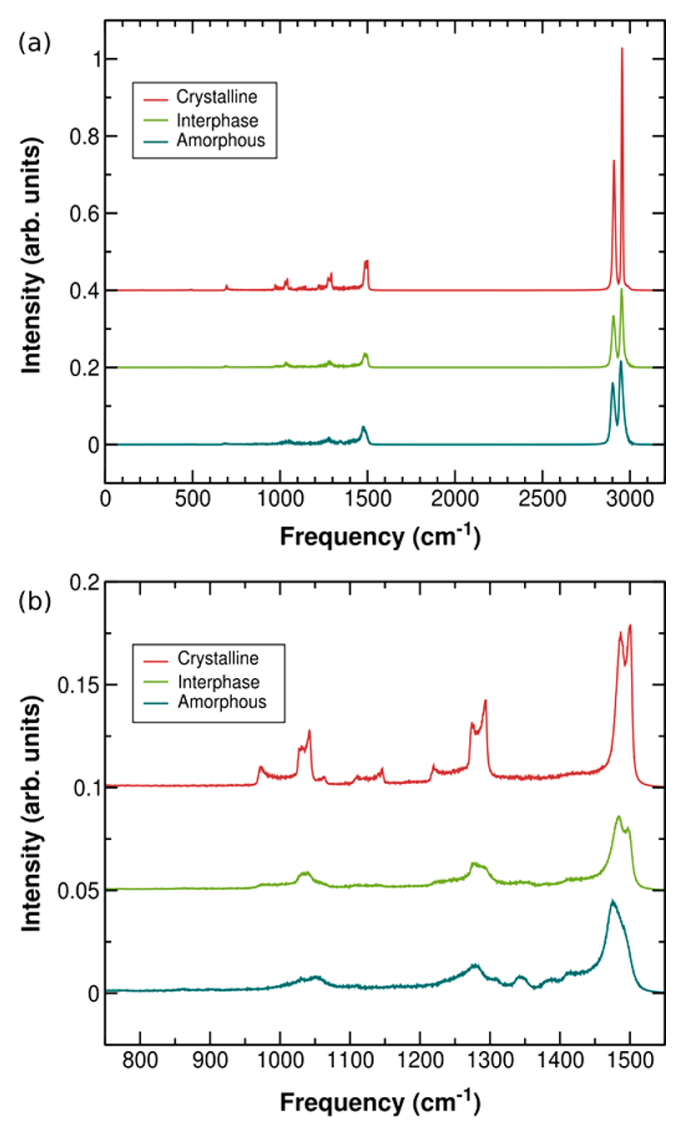

Figure 10. Vibrational spectra for the crystalline, interphase, and amorphous domains of SCPE in the EA model from 0 to $3100 \mathrm{~cm}^{-1}$ (a) and 750 to $1550 \mathrm{~cm}^{-1}$ (b) (averaged over the ten configurations). For clarity, the interphase spectra in (a) and (b) were shifted in intensity by adding 0.2 and 0.05 , respectively, while the crystalline spectra in (a) and (b) were shifted by 0.4 and 0.1 , respectively. The standard error associate with each spectrum is shown by its line width. The spectra were normalized to the maximum peak in the total vibrational spectrum. 
10b. While broadening of peaks is observed between the domains, no significant shifts in mode frequencies can be seen, in contrast to the observations for the UA model. Furthermore, some peaks in the spectrum of the crystalline domain are not present in the spectrum of the amorphous domain; most notably, the crystalline peaks near 1500, 1200, 1150, 972, and $691 \mathrm{~cm}^{-1}$ are absent from the amorphous spectrum, suggesting these peaks arise due to specific interactions in the crystal lattice. A broad peak near $1350 \mathrm{~cm}^{-1}$ with twisting character is found to be unique to the amorphous domain. A shift of the twisting band to higher frequency in the amorphous conformational state has been reported in experimental studies ${ }^{6,32}$ and by quantum mechanical calculations of $n$-alkanes. ${ }^{66}$ That the 1350 $\mathrm{cm}^{-1}$ mode stands out as a separate weak peak may be an artifact of inadequate sampling of the amorphous domain structure. Similar to the UA model, the interphase spectrum illustrates spectral features of both the crystalline and amorphous domains. The vibrational spectra of the crystalline and amorphous domains are in satisfactory agreement with the domain assignment of peaks reported for the Raman spectrum of low-density PE in the $700-1700 \mathrm{~cm}^{-1}$ region. ${ }^{32}$ However, the $\mathrm{CH}_{2}$ bending peak near $1416 \mathrm{~cm}^{-1}$, which is usually assigned to the crystalline domain, is notable absent from the calculated spectrum.

Next, we compare the simulation results to the interpretations of Strobl and Hagedorn regarding the vibrational spectrum of SCPE using the EA model. The vibrational spectra for extended-chain and melt PE reported in Figure 1 of Strobl and Hagedorn ${ }^{6}$ are qualitatively similar to the calculated spectra for the crystalline and amorphous domains, respectively. In the spectral region of the $\mathrm{C}-\mathrm{C}$ skeletal modes, peaks near 1150 and $1042 \mathrm{~cm}^{-1}$ in the spectrum of the crystalline domain are observed in reasonable agreement with those reported at 1126 and $1059 \mathrm{~cm}^{-1}$. Furthermore, the broad, $\mathrm{C}-\mathrm{C}$ stretching peak at $1042 \mathrm{~cm}^{-1}$ in the spectrum of the amorphous domain is in good agreement with the reported, broad $1080 \mathrm{~cm}^{-1}$ band observed in the spectrum of the melt. In the region of the $\mathrm{CH}_{2}$ twisting vibration, the sharp $1293 / 1276 \mathrm{~cm}^{-1}$ doublet seen in the calculated spectra of the crystalline domain is in good agreement with the reported $1292 \mathrm{~cm}^{-1}$ peak. The broad, $\mathrm{CH}_{2}$ twisting band with a maximum near $1276 \mathrm{~cm}^{-1}$ in the spectrum of the amorphous domain is similar to the reported $1303 \mathrm{~cm}^{-1}$ band in the melt signature.

Here, the spectral range of the $\mathrm{CH}_{2}$ bending vibration is discussed. Strobl and Hagedorn found evidence of a distinct interphase spectrum in this spectral region. ${ }^{6}$ In the calculated spectrum of the crystalline domain, two $\mathrm{CH}_{2}$ bending peaks were observed at 1500 and $1484 \mathrm{~cm}^{-1}$, in satisfactory agreement with an infrared-active mode around $1473 \mathrm{~cm}^{-1}$ and a Raman-active mode around $1437 \mathrm{~cm}^{-1}$. However, neither of these peaks exhibits noticeable splitting, making it difficult to verify the interpretations of Strobl and Hagedorn. Interestingly, the $\mathrm{CH}_{2}$ bending peak at $1500 \mathrm{~cm}^{-1}$ is observed clearly only in the spectra of the crystalline and interphase domains, which agrees with a typical assignment of this peak to crystalline and anisotropic phases in experimental spectra of SCPE. ${ }^{23}$ Furthermore, the relative intensities of the $\mathrm{CH}_{2}$ bending peaks at 1500 and $1484 \mathrm{~cm}^{-1}$ change in the spectrum of the interphase domain as compared to those in the crystalline domain. We found that the calculated spectrum of the interphase domain, including the change in relative intensities of the $\mathrm{CH}_{2}$ bending peaks, was adequately reproduced through a straightforward superposition of the spectra of the amorphous and crystalline domains, as demonstrated by Figure 11 (see Supporting Information for details).

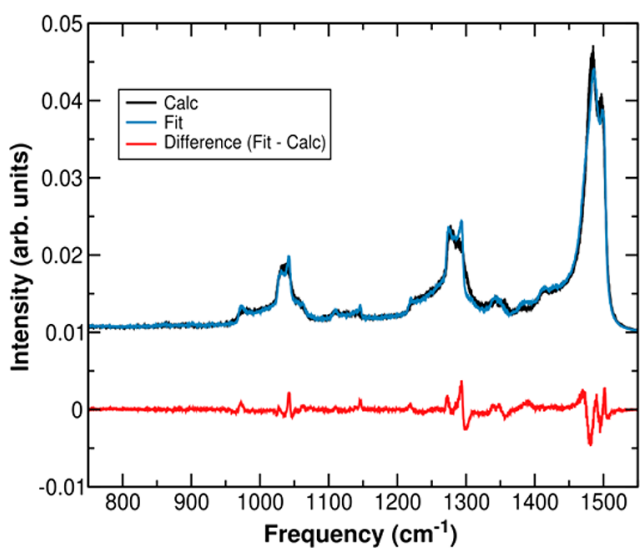

Figure 11. Comparison between the calculated interphase spectrum and the weighted superposition of the crystalline and amorphous spectra, obtained using a least-square fit to the calculated spectrum. The difference spectrum obtained by subtracting the calculated interphase spectrum from the fitted interphase is also shown. The calculated and fitted spectra were shifted by 0.01 for clarity. The line width of the calculated interphase spectrum quantifies the standard error.

We attribute the lack of observation of a distinct interphase spectrum as a consequence of the absence of the $\mathrm{CH}_{2}$ bending peak associated with the orthorhombic phase in Raman spectra. That is, the discrepancy with experiment lies in the crystal domain spectrum, not those of the noncrystalline domains. This peak was used by Strobl and Hagedorn to determine a distinct interphase spectrum, and its absence could explain why the simple superposition of crystalline and amorphous spectra is adequate for the EA model. One might be inclined to attribute the absence of the orthorhombic peak to a shortcoming of the COMPASS force field. Vibrational frequencies have been calculated previously using the COMPASS force field for orthorhombic PE; however, only infrared-active frequencies were reported. ${ }^{28}$ It is apparent from this work that the COMPASS force field does not predict the orthorhombic peak in the Raman spectrum. However, additional simulations using the $\mathrm{AMBER}^{67}$ and OPLS-AA ${ }^{68}$ force fields (see Supporting Information) also failed to reproduce the orthorhombic peak. Barrera et al. reported ab initio lattice dynamics calculation of the orthorhombic unit cell of PE using periodic density functional theory in the local density and generalized gradient approximations; they observed a peak around $1351 \mathrm{~cm}^{-1}$ and claimed good agreement with intermediate neutron scattering data. ${ }^{69}$ More recently, Galimberti et al. performed periodic density functional theory calculations of orthorhombic PE using the 6-31G(d,p) basis set and the B3LYP hybrid exchange-correlation functional with empirical correction for dispersion interactions. ${ }^{70}$ However, they calculated only the IR-active modes; to our knowledge, there are no reports of Raman spectra for orthorhombic PE calculated from first-principles at a comparable level of theory. We conclude that the missing vibration may not be related trivially to the splitting of an existing mode in the orthorhombic phase. Alternatively, the missing vibration indicates a shortcoming of Strachan's method for the purposes of calculating Raman spectra of PE. The method is sensitive to correlations in 
the positions (alternatively, the velocities or accelerations) of atomic sites, but in the absence of an explicit model for polarization, it likely errs in the estimation of intensities of peaks in the Raman spectrum.

\section{CONCLUSIONS}

In this work, we investigated the vibrational spectrum of SCPE and its distinct domains using MD simulations. We present a method for the vibrational analysis of the domains within SC polymers and subsequently used this method to analyze realistic SCPE models in both UA and EA detail. This method assigned sites in the simulations to domains based on bond orientation order, which produced interfacial widths in good agreement with previous simulation results and estimates from SAXS and Raman experiments. In the UA description, the calculated vibrational spectra were found to differ from the observed skeletal vibrations of polyethylene with the force field used in this work. We suggest modified stretching and bending force constants in the UA force field for future studies, which were demonstrated to reproduce the observed frequencies of the skeletal vibrations. In the EA description, the vibrational spectra of SCPE were in satisfactory agreement with typical infrared and Raman signatures of PE melts and crystals. However, the Raman-active $\mathrm{CH}_{2}$ bending peak associated with the orthorhombic phase was absent-an unforeseen shortcoming that is either common to a number of the currently available PE force fields or else indicative of missing information in the method used for Raman-active spectra. For the most part, the typical assignment of peaks in the Raman spectra to the components of SCPE were found to agree well with the calculated spectra of the domains. However, the spectrum of the interphase domain for the EA model was found to lack any unique spectral feature that could not be found also within the spectra of the amorphous and crystalline domains, at variance with experimental interpretations. The lack of a distinct interphase spectrum in the EA model was traced to the absence of the orthorhombic peak.

A question left unaddressed by this study is how changes in the interphase structure influences its vibrational spectrum. Crystallization conditions can have a profound impact on the structure of the noncrystalline domain. It is widely believed that the noncrystalline region in solution-crystallized material is almost completely characterized by tight loops, which might resemble the adjacent reentry model. In contrast, meltcrystallized material has a vastly different interfacial morphology, with loose loops, tails, and bridge chains that extend into a bulk amorphous region. While it has been shown that the interfacial content varies greatly between these distinct morphologies, ${ }^{24,25,32}$ the effect on its vibrational spectrum still remains unclear.

\section{ASSOCIATED CONTENT}

\section{S Supporting Information}

The Supporting Information is available free of charge on the ACS Publications website at DOI: 10.1021/acs.macromol.7b00995.

Effect of quenching procedure on the vibrational spectrum in the UA model; fitting procedure for the interphase spectrum; details on the local frame of the EA model; results of vibrational analysis with the Amber and OPLS-AA force fields; temperature dependence of vibrational spectra for orthorhombic PE crystal (PDF)

\section{AUTHOR INFORMATION}

\section{Corresponding Author}

*Tel +1 617253 0171; fax +1 617258 5766; e-mail rutledge@ mit.edu (G.C.R.).

ORCID

In-Chul Yeh: 0000-0001-9637-1700

Jan W. Andzelm: 0000-0002-2451-3770

Gregory C. Rutledge: 0000-0001-8137-1732

Notes

The authors declare no competing financial interest.

\section{ACKNOWLEDGMENTS}

Financial support of this work from US Army Research Laboratory is gratefully acknowledged.

\section{REFERENCES}

(1) Kim, J. M.; Locker, R.; Rutledge, G. C. Plastic Deformation of Semicrystalline Polyethylene under Extension, Compression, and Shear Using Molecular Dynamics Simulation. Macromolecules 2014, 47, 2515-2528.

(2) Goderis, B.; Reynaers, H.; Koch, M. H. J.; Mathot, V. B. F. Use of SAXS and Linear Correlation Functions for the Determination of the Crystallinity and Morphology of Semi-Crystalline Polymers. Application to Linear Polyethylene. J. Polym. Sci., Part B: Polym. Phys. 1999, 37, $1715-1738$

(3) Kitamaru, R.; Horii, F.; Hyon, S.-H. Proton Magnetic Resonance Studies of the Phase Structure of Bulk-Crystallized Linear Polyethylene. J. Polym. Sci., Polym. Phys. Ed. 1977, 15, 821-836.

(4) Bergmann, K.; Nawotki, K. Eine neue Interpretation der Breitlinien-Kernresonanzspektren von linearem Polyäthylen. Colloid Polym. Sci. 1967, 219, 132-144.

(5) Kotula, A. P.; Walker, A. R. H.; Migler, K. B. Raman Analysis of Bond Conformations in the Rotator State and Premelting of Normal Alkanes. Soft Matter 2016, 12, 5002-5010.

(6) Strobl, G. R.; Hagedorn, W. Raman Spectroscopic Method for Determining the Crystallinity of Polyethylene. J. Polym. Sci., Polym. Phys. Ed. 1978, 16, 1181-1193.

(7) Flory, P. J.; Yoon, D. Y.; Dill, K. A. The Interphase in Lamellar Semicrystalline Polymers. Macromolecules 1984, 17, 862-868.

(8) Ivanov, D. A.; Pop, T.; Yoon, D. Y.; Jonas, A. M. Direct Observation of Crystal-Amorphous Interphase in Lamellar Semicrystalline Poly(ethylene Terephthalate). Macromolecules 2002, 35, 9813-9818.

(9) Ghazavizadeh, A.; Rutledge, G. C.; Atai, A. A.; Ahzi, S.; Rémond, Y.; Soltani, N. Micromechanical Characterization of the Interphase Layer in Semi-Crystalline Polyethylene. J. Polym. Sci., Part B: Polym. Phys. 2013, 51, 1228-1243.

(10) Sedighiamiri, A.; Van Erp, T. B.; Peters, G. W. M.; Govaert, L. E.; van Dommelen, J. a. W. Micromechanical Modeling of the Elastic Properties of Semicrystalline Polymers: A Three-Phase Approach. J. Polym. Sci., Part B: Polym. Phys. 2010, 48, 2173-2184.

(11) Tapash, A.; DesLauriers, P. J.; White, J. L. Simple NMR Experiments Reveal the Influence of Chain Length and Chain Architecture on the Crystalline/Amorphous Interface in Polyethylenes. Macromolecules 2015, 48, 3040-3048.

(12) Brooks, B. R.; Janežič, D.; Karplus, M. Harmonic Analysis of Large Systems. I. Methodology. J. Comput. Chem. 1995, 16, 15221542.

(13) Wilson, E. B.; Decius, J. C.; Cross, P. C. Molecular Vibrations: The Theory of Infrared and Raman Spectra; Dover Publications: 1980.

(14) Strachan, A. Normal Modes and Frequencies from Covariances in Molecular Dynamics or Monte Carlo Simulations. J. Chem. Phys. 2004, 120, 1-4.

(15) Lai, P.-K.; Lin, S.-T. Internal Coordinate Density of State from Molecular Dynamics Simulation. J. Comput. Chem. 2015, 36, 507-517. 
(16) Karplus, M.; Kushick, J. N. Method for Estimating the Configurational Entropy of Macromolecules. Macromolecules 1981, 14, 325-332.

(17) Berens, P. H.; Mackay, D. H. J.; White, G. M.; Wilson, K. R. Thermodynamics and Quantum Corrections from Molecular Dynamics for Liquid Water. J. Chem. Phys. 1983, 79, 2375-2389.

(18) Lacks, D. J.; Rutledge, G. C. Simulation of the Temperature Dependence of Mechanical Properties of Polyethylene. J. Phys. Chem. 1994, 98, 1222-1231.

(19) Mizushima, S.; Simanouti, T. Raman Frequencies of N-Paraffin Molecules. J. Am. Chem. Soc. 1949, 71, 1320-1324.

(20) Boerio, F. J.; Koenig, J. L. Raman Scattering in Crystalline Polyethylene. J. Chem. Phys. 1970, 52, 3425-3431.

(21) Mutter, R.; Stille, W.; Strobl, G. Transition Regions and Surface Melting in Partially Crystalline Polyethylene: A Raman Spectroscopic Study. J. Polym. Sci., Part B: Polym. Phys. 1993, 31, 99-105.

(22) Wool, R. P.; Bretzlaff, R. S.; Li, B. Y.; Wang, C. H.; Boyd, R. H. Infrared and Raman Spectroscopy of Stressed Polyethylene. J. Polym. Sci., Part B: Polym. Phys. 1986, 24, 1039-1066.

(23) Sato, H.; Shimoyama, M.; Kamiya, T.; Amari, T.; Šašic, S.; Ninomiya, T.; Siesler, H. W.; Ozaki, Y. Raman Spectra of HighDensity, Low-Density, and Linear Low-Density Polyethylene Pellets and Prediction of Their Physical Properties by Multivariate Data Analysis. J. Appl. Polym. Sci. 2002, 86, 443-448.

(24) Glotin, M.; Mandelkern, L. A Raman Spectroscopic Study of the Morphological Structure of the Polyethylenes. Colloid Polym. Sci. 1982, 260, 182-192.

(25) Glotin, M.; Domszy, R.; Mandelkern, L. A Raman-Spectroscopic Study of Solution-Crystallized Polyethylenes. J. Polym. Sci., Polym. Phys. Ed. 1983, 21, 285-294.

(26) Krimm, S.; Liang, C. Y.; Sutherland, G. B. B. M. Infrared Spectra of High Polymers. II. Polyethylene. J. Chem. Phys. 1956, 25, 549.

(27) Schrader, B. Raman/Infrared Atlas of Organic Compounds, 2nd ed.; VCH: Weinheim, 1989.

(28) Mavrantza, I.-E.; Prentzas, D.; Mavrantzas, V. G.; Galiotis, C. Detailed Atomistic Molecular-Dynamics Simulation of the Orthorhombic Phase of Crystalline Polyethylene and Alkane Crystals. J. Chem. Phys. 2001, 115, 3937-3950.

(29) Tasumi, M.; Shimanouchi, T. Crystal Vibrations and Intermolecular Forces of Polymethylene Crystals. J. Chem. Phys. 1965, 43, 1245-1258.

(30) Dumont, D.; Henssge, E.; Fischer, D.; Bougeard, D. Simulation of Vibrational Spectra of Polymers by Molecular Dynamics Calculations. Macromol. Theory Simul. 1998, 7, 373-379.

(31) Wang, L. H.; Porter, R. S.; Stidham, H. D.; Hsu, S. L. Raman Spectroscopic Characterization of the Morphology of Polyethylene Reactor Powder. Macromolecules 1991, 24, 5535-5538.

(32) Naylor, C. C.; Meier, R. J.; Kip, B. J.; Williams, K. P. J.; Mason, S. M.; Conroy, N.; Gerrard, D. L. Raman Spectroscopy Employed for the Determination of the Intermediate Phase in Polyethylene. Macromolecules 1995, 28, 2969-2978.

(33) Lin, W.; Cossar, M.; Dang, V.; Teh, J. The Application of Raman Spectroscopy to Three-Phase Characterization of Polyethylene Crystallinity. Polym. Test. 2007, 26, 814-821.

(34) Mandelkern, L.; Alamo, R. G. Comments on Paper "Raman Spectroscopy Employed for the Determination of the Intermediate Phase in Polyethylene. Macromolecules 1995, 28, 2988-2989.

(35) He, G.; Lu, P. In Situ Confocal Micro-Raman Spectroscopic Investigation of Rearrangement Kinetics and Phase Evolution of UHMWPE during Annealing. J. Raman Spectrosc. 2015, 46, 155-160.

(36) Martín, S.; Expósito, M. T.; Vega, J. F.; Martínez-Salazar, J. Microstructure and Properties of Branched Polyethylene: Application of a Three-Phase Structural Model. J. Appl. Polym. Sci. 2012, 128, 1871-1878.

(37) Hafsia, K. B.; Ponçot, M.; Chapron, D.; Royaud, I.; Dahoun, A.; Bourson, P. A Novel Approach to Study the Isothermal and NonIsothermal Crystallization Kinetics of Poly(Ethylene Terephthalate) by Raman Spectroscopy. J. Polym. Res. 2016, 23, 1-14.
(38) in 't Veld, P. J.; Rutledge, G. C. Temperature-Dependent Elasticity of a Semicrystalline Interphase Composed of Freely Rotating Chains. Macromolecules 2003, 36, 7358-7365.

(39) Balijepalli, S.; Rutledge, G. C. Molecular Simulation of the Intercrystalline Phase of Chain Molecules. J. Chem. Phys. 1998, 109, 6523-6526.

(40) Balijepalli, S.; Rutledge, G. C. Conformational Statistics of Polymer Chains in the Interphase of Semi-Crystalline Polymers. Comput. Theor. Polym. Sci. 2000, 10, 103-113.

(41) Gautam, S.; Balijepalli, S.; Rutledge, G. C. Molecular Simulations of the Interlamellar Phase in Polymers: Effect of Chain Tilt. Macromolecules 2000, 33, 9136-9145.

(42) in 't Veld, P. J.; Hütter, M.; Rutledge, G. C. TemperatureDependent Thermal and Elastic Properties of the Interlamellar Phase of Semicrystalline Polyethylene by Molecular Simulation. Macromolecules 2006, 39, 439-447.

(43) Lee, S.; Rutledge, G. C. Plastic Deformation of Semicrystalline Polyethylene by Molecular Simulation. Macromolecules 2011, 44, 3096-3108.

(44) Rutledge, G. C. Implications of Metastability for the Crystal/ Amorphous Interface from Molecular Simulation. J. Macromol. Sci., Part B: Phys. 2002, 41, 909-922.

(45) Hütter, M.; in 't Veld, P. J.; Rutledge, G. C. Polyethylene $\{201\}$ Crystal Surface: Interface Stresses and Thermodynamics. Polymer 2006, 47, 5494-5504.

(46) Bassett, D. C.; Hodge, A. M. On the Morphology of MeltCrystallized Polyethylene I. Lamellar Profiles. Proc. R. Soc. London, Ser. A 1981, 377, 25-37.

(47) Yeh, I.-C.; Andzelm, J. W.; Rutledge, G. C. Mechanical and Structural Characterization of Semicrystalline Polyethylene under Tensile Deformation by Molecular Dynamics Simulations. Macromolecules 2015, 48, 4228-4239.

(48) Plimpton, S. Fast Parallel Algorithms for Short-Range Molecular Dynamics. J. Comput. Phys. 1995, 117, 1-19.

(49) Paul, W.; Yoon, D. Y.; Smith, G. D. An Optimized United Atom Model for Simulations of Polymethylene Melts. J. Chem. Phys. 1995, 103, 1702.

(50) Bolton, K.; Bosio; Hase, W. L.; Schneider, W. F.; Hass, K. C. Comparison of Explicit and United Atom Models for Alkane Chains Physisorbed on $\alpha$-Al2O3 (0001). J. Phys. Chem. B 1999, 103, 38853895.

(51) Wentzel, N.; Milner, S. T. Crystal and Rotator Phases of NAlkanes: A Molecular Dynamics Study. J. Chem. Phys. 2010, 132, 044901.

(52) Yi, P.; Locker, C. R.; Rutledge, G. C. Molecular Dynamics Simulation of Homogeneous Crystal Nucleation in Polyethylene. Macromolecules 2013, 46, 4723-4733.

(53) Sun, H. COMPASS: An Ab Initio Force-Field Optimized for Condensed-Phase Applications- Overview with Details on Alkane and Benzene Compounds. J. Phys. Chem. B 1998, 102, 7338-7364.

(54) Verlet, L. Computer "Experiments" on Classical Fluids. I. Thermodynamical Properties of Lennard-Jones Molecules. Phys. Rev. 1967, 159, 98-103.

(55) Eckart, C. Some Studies Concerning Rotating Axes and Polyatomic Molecules. Phys. Rev. 1935, 47, 552-558.

(56) Mavrantzas, V. G.; Theodorou, D. N. Atomistic Simulation of the Birefringence of Uniaxially Stretched Polyethylene Melts. Comput. Theor. Polym. Sci. 2000, 10, 1-13.

(57) Chilukoti, H. K.; Kikugawa, G.; Ohara, T. Self-Diffusion Coefficient and Structure of Binary N-Alkane Mixtures at the LiquidVapor Interfaces. J. Phys. Chem. B 2015, 119, 13177-13184.

(58) Stribeck, N.; Alamo, R. G.; Mandelkern, L.; Zachmann, H. G. Study of the Phase Structure of Linear Polyethylene by Means of Small-Angle X-Ray Scattering and Raman Spectroscopy. Macromolecules 1995, 28, 5029-5036.

(59) Humphrey, W.; Dalke, A.; Schulten, K. VMD: Visual Molecular Dynamics. J. Mol. Graphics 1996, 14, 33-38.

(60) Kirkwood, J. G. The Skeletal Modes of Vibration of Long Chain Molecules. J. Chem. Phys. 1939, 7, 506-509. 
(61) Liang, C. Y.; Krimm, S.; Sutherland, G. B. B. M. Infrared Spectra of High Polymers. I. Experimental Methods and General Theory. J. Chem. Phys. 1956, 25, 543-548.

(62) Davis, G. T.; Eby, R. K.; Colson, J. P. Thermal Expansion of Polyethylene Unit Cell: Effect of Lamella Thickness. J. Appl. Phys. 1970, 41, 4316-4326.

(63) Zubova, E. A.; Balabaev, N. K.; Musienko, A. I.; Gusarova, E. B.; Mazo, M. A.; Manevitch, L. I.; Berlin, A. A. Simulation of Melting in Crystalline Polyethylene. J. Chem. Phys. 2012, 136, 224906.

(64) Phillips, P. J.; Tseng, H. T. The Setting Angle of Extended Chain Linear Polyethylene. Polymer 1985, 26, 650-654.

(65) Mayo, D. W. Characteristic Frequencies of Alkanes. In Course Notes on the Interpretation of Infrared and Raman Spectra; Wyo, D., Miller, F. A., Hannah, R. W., Eds.; John Wiley \& Sons, Inc.: 2004; pp $33-72$.

(66) Tarazona, A.; Koglin, E.; Coussens, B. B.; Meier, R. J. Conformational Dependence of Raman Frequencies and Intensities in Alkanes and Polyethylene. Vib. Spectrosc. 1997, 14, 159-170.

(67) Cornell, W. D.; Cieplak, P.; Bayly, C. I.; Gould, I. R.; Merz, K. M.; Ferguson, D. M.; Spellmeyer, D. C.; Fox, T.; Caldwell, J. W.; Kollman, P. A. A Second Generation Force Field for the Simulation of Proteins, Nucleic Acids, and Organic Molecules. J. Am. Chem. Soc. 1995, 117, 5179-5197.

(68) Jorgensen, W. L.; Maxwell, D. S.; Tirado-Rives, J. Development and Testing of the OPLS All-Atom Force Field on Conformational Energetics and Properties of Organic Liquids. J. Am. Chem. Soc. 1996, $118,11225-11236$.

(69) Barrera, G. D.; Parker, S. F.; Ramirez-Cuesta, A. J.; Mitchell, P. C. H. The Vibrational Spectrum and Ultimate Modulus of Polyethylene. Macromolecules 2006, 39, 2683-2690.

(70) Galimberti, D.; Milani, A.; Maschio, L.; Castiglioni, C. Intermolecular Modulation of IR Intensities in the Solid State. The Role of Weak Interactions in Polyethylene Crystal: A Computational DFT Study. J. Chem. Phys. 2016, 145, 144901. 